\title{
An integrated system for scheduling of processing and assembly operations with fuzzy operation time and fuzzy delivery time
}

\author{
Yang, M.S. ${ }^{a}{ }^{*}$, Ba, L. ${ }^{a}{ }^{*}$, Zheng, H.Y. ${ }^{a}$, Liu, Y. ${ }^{a}$, Wang, X.F. ${ }^{a}$, He, J.Z. ${ }^{a}$, Li, Y. ${ }^{a}$ \\ ${ }^{a}$ School of Mechanical and Precision Instrument Engineering, Xi'an University of Technology, Xi'an, P.R. China
}

\begin{abstract}
A B S T RACT
This paper integrates the processing scheduling with assembly scheduling, aiming to satisfy the requirem ents for jus t-in-time (JIT) production. Considering the uncertainty of time factors in actual production, the operation time of the jobs were represented as triangular fuzzy numbers and the delivery time of the final product as trapezoidal fuzzy numbers. An extended job-shop scheduling problem (JSP) considering above factors was proposed in this paper. A mathematical model was established for processing and assembly scheduling, aiming to achieve the mean satisfaction degree on delivery time. In light of the complexity of the problem, a genetic algorithm (GA) was designed to realize the fuzzy integrated optimization of processing and assembly under uncertainty. The proposed algorithm includes selection, crossover, mutation operations, and reflects the spirits of two-section real number encoding and elite protection strategy. Each part of the GA was designed in detail. Finally, the proposed model and algorithm were verified thro ugh a case study on processing and assembly scheduling. The model enjoys high practical value by taking the customer satisfaction of the delivery period as the main goal. The results show that our scheduling strategy mirrors the actual production situation and provides a good reference for JSP scheduling under multiple uncertainties. The best solution obtained by our model is more feasible than basic JSP in real production environment.
\end{abstract}

\author{
ARTICLE INFO \\ Keywords: \\ Integrated scheduling; \\ Uncertainty; \\ Fuzzy operation time; \\ Fuzzy delivery time; \\ Genetic algorithm (GA) \\ *Corresponding author: \\ yangmingshun@xaut.edu.cn \\ (Yang, M.S.) \\ xautbali@163.com \\ (Ba, L.) \\ Article history: \\ Received 18 April 2019 \\ Revised 8 September 2019 \\ Accepted 12 September 2019
}

\section{References}

[1] Nagata, Y., Ono, I. (2018). A guided local search with iterative ejections of bottleneck operations for the job shop scheduling problem, Computers \& Operations Research, Vol. 90, 60-71, doi: 10.1016/j.cor.2017.09.017.

[2] Sharma, N., Sharma, H., Sharma, A. (2018). Beer froth artificial bee colony algorithm for job-shop scheduling problem, Applied Soft Computing, Vol. 68, 507-524, doi: 10.1016/i.asoc.2018.04.001.

[3] Bierwirth, C., Kuhpfahl, J. (2017). Extended GRASP for the job shop scheduling problem with total weighted tardiness objective, European Journal of Operational Research, Vol. 261, No. 3, 835-848, doi: 10.1016/i.ejor.2017. $\underline{03.030 .}$

[4] Piroozfard, H., Wong, K.Y., Asl, A.D. (2017). An improved biogeography-based optimization for achi eving optimal job shop scheduling solutions, Procedia Computer Science, Vol. 115, 30-38, doi: 10.1016/i.procs.2017.09.073.

[5] Akram, K., Kamal, K., Zeb, A. (2016). Fast simulated annealing hybridized with quenching for solving job shop scheduling problem, Applied Soft Computing, Vol. 49, 510-523, doi: 10.1016/i.asoc.2016.08.037.

[6] Hu, H.X., Lei, W.X., Gao, X., Zhang, Y. (2018). Job-shop scheduling problem based on improved cuckoo search algorithm, International Journal of Simulation Modelling, Vol. 17, No. 2, 337-346, doi: 10.2507/IJSIMM17(2)C08.

[7] Modrák, V., Pandian, R.S. (2010). Flow shop scheduling algorithm to minimize completion time for n-jobs mmachines problem, Tehnički Vjesnik - Technical Gazette, Vol.17, No. 3, 273-278. 
[8] Lin, J. (2019). Backtracking search bas ed hyper-heuristic for the flexible job-shop scheduling problem wi th fuzzy processing time, Engineering Applications of Artificial Intelligence, Vol. 77, 186-196, doi: 10.1016/i.engappai. $\underline{2018.10 .008}$

[9] Shen, L., Dauzère-Pérès, S., Neufeld, J.S. (2018). Solving the flexible job shop scheduling problem with sequencedependent setup times, European Journal of Operational Research, Vol. 265, No. 2, 503-516, doi: 10.1016/j.ejor. 2017.08.021.

[10] Kato, E.R.R., Aranha, G.D.A., Tsunaki, R.H. (2018). A new approach to solve the flexible job shop problem based on a hybrid particle swarm optimization and random-restart hill climbing, Computers \& Industrial Engineering, Vol. 125, 178-189, doi: 10.1016/i.cie.2018.08.022.

[11] Wu, X., Sun, Y. (2018). A green scheduling algorithm for flexible job shop with energy-saving measures, Journal of Cleaner Production, Vol. 172, 3249-3264, doi: 10.1016/i.jclepro.2017.10.342.

[12] Yang, X.P., Gao, X.L. (2018). Optimization of dynamic and multi-objective flexible job-shop scheduling based on parallel hybrid algorithm, International Journal of Simulation Modelling, Vol. 17, No. 4, 724-733, doi: 10.2507/ IISIMM17(4)CO19.

[13] Seng, D.W., Li, J.W., Fang, X.J., Zhang, X.F., Chen, J. (2018). Low-carbon flexible job-shop scheduling based on improved no ndomi nated sorting genetic al gori thm-II, International Journal of Simulation Modelling, Vol. 17, No. 4, 712-723, doi: 10.2507/IISIMM17(4)CO18.

[14] Nidhiry, N.M., Saravanan, R. (2014). Scheduling optimization of a flexible manufacturing system using a modified NSGA-II algorithm, Advances in Production Engineering \& Management, Vol. 9, No. 3, 139-151, doi: 10.14743/ apem2014.3.183.

[15] Xu, H., Bao, Z.R., Zhang, T. (2017). Solving dual flexible job-shop scheduling problem using a Bat Algo rithm, Advances in Production Engineering \& Management, Vol.12, No. 1, 5-16, doi: 10.14743/apem2017.1.235.

[16] Yazdani, M., Aleti, A., Khalili, S.M., Jolai, F. (2017). Optimizing the sum of maximum earliness and tardiness of the job shop scheduling problem, Computers \& Industrial Engineering, Vol. 107, 12-24, doi: 10.1016/i.cie.2017. $\underline{02.019}$.

[17] Chaouch, I., Driss, O.B., Ghedira, K. (2017). A modified ant colony optimization algo rithm for the distributed job shop scheduling problem, Procedia Computer Science, Vol.112, 296-305, doi: 10.1016/i.procs.2017. 08.267.

[18] Kurdi, M. (2017). An improved island model memetic algorithm with a new cooperation phase for multiobjective job shop scheduling problem, Computers \& Industrial Engineering, Vol. 111, 183-201, doi: 10.1016/ j.cie.2017.07.021.

[19] Shahrabi, J., Adibi, M.A., Mahootchi, M. (2017). A reinforcement learning approach to parameter estimation in dynamic job shop scheduling, Computers \& Ind ustrial Engineering, Vol. 110, 75-82, doi: 10.1016/i.cie.2017.05. $\underline{026}$.

[20] Kundakcı, N., Kulak, O. (2016). Hybrid genetic al gorithms for minimizing makespan in dynamic job shop scheduling problem, Computers \& Industrial Engineering, Vol. 96, 31-51, doi: 10.1016/i.cie.2016.03.011.

[21] Kuhpfahl, J., Bierwirth, C. (2016). A study on local search neighborhoods for the job shop scheduling problem with total weighted tardiness objective, Computers \& Operations Research, Vol. 66, 44-57, doi: 10.1016/ j.cor.2015.07.011.

[22] Zhong, Q., Yang, H., Tang, T. (2018). Optimization algorithm simulation for dual-resource constrained job-shop scheduling, International Journal of Simulation Modelling, Vol. 17, No. 1, 147-158, doi: 10.2507/ISSIMM17(1)CO2.

[23] Haider, A., Mirza, J. (2015). An implem entation of lean scheduling in a job shop enviro nm ent, Advances in Production Engineering \& Management, Vol. 10, No. 1, 5-17, doi: 10.14743/apem2015.1.188.

[24] Chaudhry, I.A., Usman, M. (2017). Integrated process planning and scheduling using genetic algorithms, Tehnički Vjesnik - Technical Gazette, Vol. 24, No. 5, 1401-1409, doi: 10.17559/TV-20151121212910.

[25] Yu, H. (2018). Numerical simulation of European option payoff based on stochastic differential delay equations, Mathematical Modelling of Engineering Problems, Vol. 5, No. 2, 102-107, doi: 10.18280/mmep.050207.

[26] Song, S.L. (2018). Application of gray prediction and linear programming model in economic management, Mathematical Modelling of Engineering Problems, Vol. 5, No. 1, 46-50, doi: 10.18280/mmep.050107.

[27] Kueh, S.M., Kuok, K.K. (2018). Forec asting long term precipitation using cuckoo search optimization neural network models, Environmental Engineering and Management Journal, Vol. 17, No. 6, 1283-1291, doi: 10.30638/ eemj.2018.127. 


\title{
Integrirani sistem za razporejanje izdelave in montaže z mehkim časom izdelave in mehkim dobavnim rokom
}

\author{
Yang, M.S. ${ }^{a,}{ }^{*}$, Ba, L. ${ }^{a},{ }^{*}$, Zheng, H.Y. ${ }^{a}$, Liu, Y. ${ }^{a}$, Wang, X.F. ${ }^{a}$, He, J.Z. ${ }^{a}$, Li, Y. ${ }^{a}$ \\ ${ }^{a}$ School of Mechanical and Precision Instrument Engineering, Xi'an University of Technology, Xi'an, P.R. China
}

\section{POVZETEK}

Ta članek združuje razporejanje izdelave $\mathrm{z}$ razporejanjem montaže, da bi zadovoljili zahtevam po sprotni proizvodnji (JIT). Če upoštevamo negotovost časovnih dejavnikov v dejanski proizvodnji, je bil čas izdelave predstavljen kot trikotno mehko število, dobavni rok končnega izdelka pa kot trapezno mehko število. V tem prispevku je bil, glede na zgoraj navedene dejavnike, predlagan razširjen problem terminiranja proizvodnje po naročilu (JSP). Vzpostavljen je bil matematični model za razporejanje izdelave in montaže, s ciljem doseči povprečno stopnjo zadovoljstva z ozirom na čas dobave. Glede na kompleksnost problema je bil zasnovan genetski algoritem (GA), ki omogoča mehko integrirano optimizacijo izdelave in montaže $\mathrm{z}$ upoštevanjem negotovostji. Predlagani algoritem vključuje mehanizme izbire, križanja, mutacije, dvosektorskega realnega številčnega kodiranja in ohranjanja elitistične strategije. Vsak del GA je bil zasnovan podrobno. Nazadnje sta bila predlagani model in algoritem preverjena s študijo primera razporejanja izdelava in montaže. Model ima visoko praktično vrednost, saj kot glavni cilj upošteva zadovoljstvo kupcev z ozirom na čas dobave. Rezultati kažejo, da naša strategija razporejanja odraža dejanske proizvodne razmere in nudi dobro referenco za JSP z več negotovostmi. Najboljša rešitev, ki jo dobimo z našim modelom, je v realnem proizvodnem okolju izvedljivejša od rešitve osnovnega JSP.
\end{abstract}

\section{PODATKI O ČLANKU}

Ključne besede:

Integrirano razporejanje;

Negotovost;

Mehki čas izdelave;

Mehki dobavni rok;

Genetski algoritem (GA)

*Kontaktna oseba:

yangmingshun@xaut.edu.cn

(Yang, M.S.)

xautbali@163.com

(Ba, L.)

Zgodovina članka:

Prejet 18. aprila 2019

Popravljen 8. septembra 2019

Sprejet 12. septembra 2019 